\title{
Philosophical Geometers and Geometrical Philosophers
}

\author{
Chris Smeenk \\ Western University
}

\begin{abstract}
[S]ince an exact science of [colors] seems to be one of the most difficult things that Philosophy is in need of, I hope to show - as it were, by my example - how valuable mathematics is in natural Philosophy. I therefore urge geometers to investigate nature more rigorously, and those devoted to natural science to learn geometry first. Hence the former shall not entirely spend their time in speculations of no value to human life, nor shall the latter, while working assiduously with an absurd method, perpetually fail to reach their goal. But truly with the help of philosophical geometers and geometrical philosophers, instead of the conjectures and probabilities that are blazoned about everywhere, we shall finally achieve a science of nature supported by the highest evidence. (Optical Papers, 86-89)
\end{abstract}

\section{Introduction}

It is common to regard Newton as the apotheosis of mathematized natural philosophy in the seventeenth century. For example, the Principia Mathematica is the culmination of Dijksterhuis's grand narrative of mechanization, ${ }^{1}$ marking the transition to a thorough mathematization of science. Accounts like this reflect Newton's transformative contributions to natural philosophy and the central role of mathematics in his achievements. Newton frequently characterized his methodology, as in the epigraph from 1672, as distinctive and capable of achieving greater evidential support than that of his contemporaries, due to its mathematical character. Those guilty of blazoning about mere conjectures are presumably mechanical philosophers, including those working in the Cartesian tradition as well as members of the Royal Society such as Hooke and Boyle. The remark cannot be dismissed as merely reflecting the brashness of youth, the overly dogmatic stance of a 27 year old that became more moderate with age. The emphasis on the role of mathematics in achieving certainty in natural philosophy, as well as the contrast with the errant ways of others, recurs with variations in methodological remarks throughout Newton's career.

Newton's pronouncements reflect a striking position regarding the role of mathematics in natural philosophy. We can give an initial characterization of his position by considering two questions central to seventeenth century debates about the applicability of mathematics. First, how are we to understand the distinctive universality and necessity of mathematical reasoning? One common way to preserve the demonstrative character of mathematics was to restrict its domain, as far as possible, to pure abstractions. The subject matter of mathematics is then taken to be abstracted from the changeable natural world, consisting of quantity and magnitude themselves rather than the objects bearing quantifiable properties. Yet restricting the domain in this way leaves it difficult to see how mathematics relates to natural phenomena. How could the book of nature be

1. E. J. Dijksterhius, The Mechanization of the World Picture, Paperback (1986), trans. C. Dikshoorn (Princeton University Press, 1961). 
written in a language of pure abstractions? Second, what is the proper role of mathematical reasoning in natural philosophy? Many followed Aristotle in consigning mathematics to a subordinate role. On this view, mathematical demonstrations do not contribute to scientific knowledge because they do not proceed from causes. The demand for such demonstrations was difficult to satisfy in mathematics, especially for those who rejected formal causality. Mathematics could not fulfill the main aim of natural philosophy, namely to provide demonstrations reflecting the essences of things and nature's causal order. A related concern was also pressing for the mechanical philosophers: a merely mathematical demonstration fails to provide an intelligible mechanical explanation. For advocates of this line of thought, the very title of Newton's masterpiece, Philosophiae Naturalis Principia Mathematica, would have been extremely perplexing: how could natural philosophy be based on mathematical principles?

Newton's "mathematico-physical" approach, as Halley characterized the Principia in his ode, reflected an alternative line of thought in seventeenth century debates on the status of mathematics. In particular, Thomas Hobbes and Isaac Barrow, Newton's predecessor as Lucasian Chair, both held that the demonstrative character of mathematics does not require a restriction to quantity regarded abstractly. Hobbes's materialist mathematics left no place for a distinction between "pure" and "applied" mathematics: real bodies and their properties were the proper subject matter of mathematics. Hobbes further argued that demonstrations proceeding from the properties of real bodies satisfied demands for knowledge based on causes. For Hobbes the demonstrative character of mathematics, far from conflicting with the inclusion of physical concepts regarding body and motion in the definitions, actually resulted from it. There was then no reason to regard mathematics as inherently subordinate, unable to advance the aims of natural philosophy. Like Hobbes, Barrow offered a defense of the scientific status of mathematics, but with geometric demonstrations satisfying a kind of formal causality. He also collapsed the distinction between "pure" geometry and physics. Barrow regarded geometrical objects as generated through motion, leaving no gap between space and continuous magnitude, studied geometrically, and motions in real space, studied in physics. Newton developed a position similar to that of Barrow and Hobbes, although I will not here trace their influence in detail.

My aim is to articulate Newton's position regarding the mathematization of nature. This is challenging because Newton, unlike Hobbes and Barrow, never stated a systematic philosophy of mathematics. Yet it is crucial to articulate Newton's position given his enormously creative and influential contributions to mathematics and natural philosophy. On my reading, Newton regards the traditional response to the first question, which takes mathematical concepts to apply to abstract rather than material entities, to be deeply mistaken. Newton holds instead that "rational mechanics" offers as exact a description of material objects as the description of abstracta allegedly provided by mathematics. Yet he does not take this exactness to be directly revealed in experience. An exact description underlies experience, but the underlying quantitative description can only be reconstructed from observations within an appropriate framework.

Regarding the second question, the aim of natural philosophy is taken to be the articulation of the appropriate framework for uncovering fundamental quantites and regularities. Newton develops an account of force in the Principia that provides such a framework, in that it underwrites theorems relating properties of observed motions to properties of a given force law (and vice versa). Making a convincing case in favor of the force of gravity to his contemporaries required overcoming opposition to this new approach to natural philosophy, which placed forces characterized quantitatively at the center of investigation. It is also not clear how to bring to bear the traditional demand that a demonstration must proceed from causes. Mathematics provides an inferential framework for understanding the forces relevant to phenomena, and for extracting an exact quantitative description of motion from inexact experience. While it is certainly appropriate to ask whether this 
approach has succeeded in discovering the true forces of nature from complex phenomena, it is less clear that there is a valid remnant of the Aristotelian demand for causes.

The paper will develop and defend this reading of Newton's position as follows. The next section focuses on Newton's discussions of the nature and status of geometry in the preface to the Principia and related texts, in which he argues that mechanics and geometry do not differ, as was traditionally assumed, in terms of their objects of study. Rather than treating geometry as a self-sufficient inquiry focused on distinctive, abstract entities, Newton characterized it as relying upon rational mechanics for an account of the generation of geometrical objects, and differing primarily in its more restricted scope. The intelligibility of the objects of study for both geometry and rational mechanics depends on understanding how the object is generated; geometry describes objects as generated from a restricted set of allowed constructions, whereas rational mechanics studies real motions produced by forces. Section 3 relates Newton's views on geometry to the aims of natural philosophy and how he pursued these in the Principia. Natural philosophy led Newton to extend geometry in two different senses: first, by rejecting Descartes' restrictions to particular types of curves, and, second, by including reasoning using first and last ratios to allow treatment of instantaneous quantities. This extension was required to deal with the complexity of real phenomena. Newton's sophisticated methodology, briefly described here, aimed to extract the underlying forces from a study of phenomena. Following these discussions of the nature of geometry and Newton's reformulation of the aims of natural philosophy such that mathematical principles play a central role, Section 4 turns to Newton's views on the certainty of mathematics.

\section{Geometry and Mechanics}

The Principia begins with a discussion of the relationship between geometry and mechanics, in the Author's preface:

Geometry does not teach how to describe these straight lines and circles, but postulates such a description. For geometry postulates that a beginner has learned to describe lines and circles exactly before he has reached the threshold of geometry, and then it teaches how problems are solved by these operations. To describe straight lines and to describe circles are problems, but not problems in geometry. Geometry postulates the solution of these problems from mechanics and teaches the use of the problems thus solved. And geometry can boast that with so few principles obtained from other fields, it can do so much. Therefore, geometry is founded on mechanical practice and is nothing other than that part of universal mechanics which reduces the art of measuring to exact proportions and demonstrations. But since the manual arts are applied especially to making bodies move, geometry is commonly used in reference to magnitude, and mechanics in reference to motion. In this sense, rational mechanics will be the science, expressed in exact propositions and demonstrations, of the motions that result from any forces whatever and of the forces that are required for any motions whatever. [...] [S] ince we are concerned with natural philosophy [...] we concentrate on aspects of gravity, levity, elastic forces, resistance of fluids, and forces of this sort, whether attractive or repulsive. And therefore our present work sets forth mathematical principles of natural philosophy. (Principia, 381-382.) ${ }^{2}$

2. References to the Principia are to Isaac Newton, The Principia, Mathematical Principles of Natural Philosophy: A New Translation, trans. I. Bernard Cohen and Anne Whitman (Berkeley, California: University of California Press, 1999 [1726]), and MP n abbreviates Volume $\mathrm{n}$ of D.T. Whiteside, ed., The Mathematical Papers of Isaac Newton, vol. 1-8 (Cambridge, London: Cambridge University Press, 1967-81). 
There are several intriguing claims in this passage, which Newton explored at greater length in unpublished manuscripts. ${ }^{3}$ Although epistemology of geometry is not the central focus of an extended treatise, these texts suffice to elucidate aspects of his position and how it contrasts with views of his contemporaries.

Geometry is founded on mechanical practice in the sense that it is turns to mechanics for the construction or generation of the objects used in geometrical reasoning. Newton adopts a kinematic conception of geometry, in which an object such as a curve is understood in terms of how it can be generated by motion. One can impose restrictions on the permissible ways of generating curves and other geometric objects, such as using only a compass and straightedge. But, as Newton emphasizes at the start of the passage, the repertoire of constructions is not the subject matter of, nor is it fixed by, geometry itself: mechanics rather than geometry determines the permissible constructions. This repertoire includes far more than straightedge and compass constructions. Newton's rejection of constraints on the methods used to generate a curve is more explicit in the Geometria:

[W]e are free to describe them [plane figures] by moving rulers around, using optical rays, taut threads, compasses, the angle given in a circumference, points separately ascertained, the unfettered motion of a careful hand, or finally any mechanical means whatever. Geometry makes the unique demand that they are described exactly. (MP 7: 289)

Newton's only constraint on the generation of curves is that their construction must be "exact."

Newton's emphasis on "exactness" counters a common view that the subject matter of mechanics cannot be described with sufficient precision to be studied geometrically. He rejects the "common belief" that "nothing could possibly be mechanical and at the same time exact" as a "stupid one" (MP 7: 289). The common view mistakenly treats the flaws of particular instances of manual generation of curves as a general failing of mechanics. The appropriate contrast, Newton argues in the Geometria, concerns the different aims of mechanics and geometry: mechanics concerns the form and generation of continuous magnitudes, whereas geometry is the science of measurement of such quantities. What Newton means by the exactness characteristic of both can be discerned in his formulation of new set of postulates for geometry; the third postulate allows a quite general construction which "has a kinship with mechanical description by moving rulers". The third postulate states:

To draw any line on which there shall always fall a point which is given according to a precise rule by drawing from points through points lines congruent to given ones. (MP 7: 389)

The demand for exactness is reflected in this requirement that there is a precise rule for generating the curve. Newton goes on to discuss how this postulate licenses the construction of a wide variety of curves.

Newton implicitly rejects the criteria of intelligibility proposed in Descartes' Géométrie, one of the texts that had inspired his early work in mathematics. ${ }^{4}$ Descartes's criteria was formulated in

3. The unpublished manuscripts include, in particular, the Geometriae (ca. 1692, published in MP 7), a long treatise on geometry Newton undertook in the 1690s, whose opening discussion amplifies the themes of the preface, as well as shorter fragments. My comments here are indebted to the discussion in N. Guicciardini, Isaac Newton on mathematical certainty and method (The MIT Press, 2009), Chapters 13-14 (subsequent references to Guicciardini are to this book), and M. Domski, "The constructible and the intelligible in Newton's philosophy of geometry," Philosophy of Science 70, no. 5 (2003): 1114-1124; M. Domski, "Newton as Historically-Minded Philosopher," in Discourse on a New Method: Reinvigorating the Marriage of History and Philosophy of Science, ed. M. Domski and M. Dickson (Open Court Pub Co, 2010), 65 .

4. See Domski, in "The constructible and the intelligible in Newton's philosophy of geometry". Newton also criticizes the Cartesian, algebraic approach to the classification of curves in the Appendix of Arithmetica Universalis. 
terms of the means of generating a curve as well as its algebraic representation, with the scope of geometry limited to curves generated via specific generalizations of compass and straight-edge constructions, or, expressed algebraically, to curves represented by closed polynomials. (Descartes hoped to prove that these two characterizations were equivalent, but failed. ${ }^{5}$ ) This excluded "mechanical" or "transcendental" curves from consideration, for example the logarithmic spiral and cycloid. Newton rejected both grounds Descartes gave for regarding such curves as falling outside the scope of geometry, and studied the properties of non-mechanical curves in his early mathematical research in the 1660s as well as the Geometria. Newton discovered how to represent this more general class of curves algebraically via an infinite series expansion (Guicciardini, Chapter 7). We will see the significance of the resulting broader scope for geometry in the next section.

Newton regards "rational mechanics" as the exact science of the generation of the motions of real bodies, due to the combination of inertia and forces acting on them. Shifting from "manual powers" to "forces" does not alter the idea that the intelligibility of a given curve depends on understanding how it is generated, given an appropriate conception of force. One of Newton's achievements was the recognition of how much was required in clarifying the conceptions of inertia and force, in order to undertake the project of determining the true motion of bodies. ${ }^{6}$ Unlike his contemporaries, he argued that providing an account of the dynamics governing motion could not be adequately founded on geometrical relationships among bodies alone, but required in addition an appeal to "absolute" structures (namely, intervals of spatial distance and temporal duration, and a way of identifying locations over time). These structures underwrite a contrast between inertial (moving in a straight line at uniform velocity) and non-inertial motion. With this contrast in place, it is in principle possible to consider a physical trajectory as generated by the net force acting on a body in much the same manner as the geometry student manually producing a curve. And, importantly, it is possible to draw inferences in the opposite direction as well: that is, given the trajectory, to determine the net force that would produce the required motion.

Geometry and rational mechanics are thus both exact sciences with a common subject matter: geometry measures the properties of objects whose generation is described by mechanics. It seems more apt to call this physicalizing geometry rather than mathematizing the study of motion. Our sensible experience of the geometrical properties of objects and their trajectories may be vague or inexact. The trajectories of real bodies are still suitable objects of study for mechanics and geometry, even though they are only accessible via a combination of observation and calculation. The tension between the exactness of geometry and mechanics and the character of sensations is resolved by taking geometry to apply directly to physical objects, whose geometrical properties are immanent in sensation rather than directly apparent. Since the properties are not ascribed to abstract mathematical entities with a distinctive ontological status, there is no place for a worry to arise regarding how mathematical entities can stand in relation to, or represent, physical objects.

Newton's account of the nature of geometry stands in stark contrast with the Cartesian tradition, as well as the traditional Aristotelian account of pure and mixed mathematics. Newton's position was not unprecedented, and it is particularly useful to compare Newton's position with

5. H.J.M. Bos, Redefining geometrical exactness: Descartes' transformation of the early modern concept of construction (New York: Springer Verlag, 2001).

6. Newton takes up this question in "De Gravitatione" (discussed in $\S 4$ below) and the famous Scholium to the definitions (see Howard Stein, "Newtonian space-time," Texas Quarterly 10 [1967]: 174-200; John S. Earman, World Enough and Space-Time: Absolute versus Rational Theories of Space and Time [Cambridge, MA: MIT Press, 1989]; R. Rynasiewicz, "By their properties, causes and effects: Newton's scholium on time, space, place and motion-I. The text," Studies In History and Philosophy of Science Part A 26, no. 1 [1995]: 133-153; R. DiSalle, Understanding space-time: The philosophical development of physics from Newton to Einstein [Cambridge: Cambridge Univ Press, 2006]). 
that of Barrow. ${ }^{7}$ Barrow's Geometrical Lectures highlighted the utility of treating curves in terms of generating motions rather than as a collection of points. Several of Barrow's predecessors solved problems such as that of finding tangents and areas of a curve based on such a kinematic conception, but Barrow treated the generation of curves by motion as the appropriate foundation for geometry rather than simply a useful heuristic. Barrow argued further that the definition of curves in terms of their generating motions satisfied traditional demands for causal arguments, and regarded geometry as the most fundamental branch of mathematics because it deals directly with magnitudes generated by motion. ${ }^{8}$

Newton was almost certainly influenced by Barrow's defense of the scientific status of mathematics in his earlier Mathematical Lectures. Barrow held that mathematical demonstrations satisfy a version of formal causality. ${ }^{9}$ He also addressed a problem facing any empiricist epistemology of geometry, namely how sense experience relates to geometric reasoning. Barrow characterized the contribution of sensation as limited to establishing that geometrical postulates reflect a real possibility. We can see that a straight line is a real possibility by considering an actual line, and further recognizing that there is no obstacle to making it straighter. Sensation does not provide an inductive base for geometrical arguments, but instead establishes that geometrical postulates are not empty or vacuous. Against the view that geometrical objects are merely mental entities, Barrow asserts that "all imaginable Geometrical Figures are really inherent in every Particle of Matter," even if they are inaccessible to the senses, ${ }^{10}$ just as the statue is in a block of marble, waiting to be uncovered by the sculptor's chisel. Barrow further rejected the traditional distinction between pure and mixed mathematics. It was common, following Aristotle, to regard pure mathematics as restricted to the study of quantities abstracted from material objects, whereas mixed mathematics applied to the mathematical properties imperfectly instantiated by sensible objects. Since for Barrow the instantiation of geometrical properties in sensible objects underwrites their intelligibility, this contrast makes no sense. Barrow collapsed this distinction by taking geometry to apply directly to material objects, even though their exact geometrical properties are not immediately revealed in sensation.

The Principia's preface echoes this position, but Barrow's effort to ground mathematics solely on geometry, as he conceived it, is incompatible with Newton's mathematical practice. By the time Barrow presented his Geometrical Lectures in 1668, Newton had made many strikingly innovative discoveries in what he called the fluxional analysis of curves. Newton's first treatise, "To Resolve Problems by Motion" (1666), began like Barrow with a kinematic conception of curves. Newton

7. The comparison between Barrow and Newton, which I return to in $\S 4$ below, is explored in much greater depth by Katherine Dunlop, "What geometry postulates: Newton and Barrow on the relationship of mathematics to nature," Interpreting Newton: Critical essays (2012): 69-101, "The mathematical form of measurement and the argument for Proposition I in Newton's Principia," Synthese 186, no. 1 (2012): 191-229. See Guicciardini, Chapter 8 for a detailed contrast between Barrow's mathematics and Newton's fluxional analysis, and Michael S. Mahoney, "Barrow's mathematics: between ancients and moderns," Before Newton: The Life and Times of Isaac Barrow (1990): 179-249; Ian Stewart, "Mathematics as Philosophy: Proclus and Barrow," Dionysius 18 (2000): 151-181, for further discussion of Barrow's mathematics.

8. Hobbes defended a similar view, namely that the scientific status of mathematics depended on the generation of geometrical magnitudes by motion, in an extended polemic with John Wallis, described with a clear account of the philosophy of mathematics involved by Jesseph, in Squaring the circle: the war between Hobbes and Wallis (Chicago: University of Chicago Press, 1999). Newton studied De Corpore closely in his student years, but I am not aware of any evidence regarding his assessment of the Hobbes - Wallis exchange.

9. Barrow was responding to debates regarding the scientific character of mathematical demonstrations going back to Piccolimini's 1547 treatise (see Mancosu, in Philosophy of mathematics and mathematical practice in the seventeenth century (Oxford University Press, 1996); considered in the context of these debates, Barrow advocates an unorthodox account of formal causality.

10. Isaac Barrow, The Usefulness of Mathematical Learning Explained and Demonstrated: Being Mathematical Lectures Read in the Publick Schools at the University of Cambridge (London: Stephen Austen, 1734), 76. 
appeals to the continuity of motion generating a curve to justify the use of limiting procedures. Yet Newton's development of the calculus depended on combining a kinematic conception with ideas from algebra foreign to Barrow's approach. In particular, Newton discovered a generalization of the binomial theorem to non-integer exponents, which allowed him to treat curves such as the logarithmic spiral using an infinite series expansion. This result was inspired by Wallis's Arithmetica Infinitorum, and Newton's mature 1671 treatise develops fluxional analysis based on a kinematic approach to curves used in concert with algebraic techniques. ${ }^{11}$

The importance of these algebraic techniques is largely hidden from view in the Principia, which Newton wrote in a synthetic, geometric style. Newton's wide range of mathematical techniques peeks through the chinks in the armor of synthetic geometry. The types of problems Newton handled earlier using fluxional analysis are treated in the Principia based on a geometrical treatment of limits (described briefly below). Newton had several reasons for adopting a geometrical style. ${ }^{12}$ For many of the problems in the Principia, geometrical methods may have been the most efficient and direct calculational tool. But it is also the case that Newton's views regarding mathematics shifted, as he developed admiration for Greek geometry based on studying Pappus and Apollonius in the 1670s. While I do not have the space to explore the issue fully here, it is clear that the Principia's proofs required combining geometrical reasoning (suitably extended) with a variety of algebraic techniques. Insights from fluxional analysis are crucial in a number of places, for example the proofs in Section 8 and in various cases where Newton states the area under a curve without specifying his method for finding it. Book II includes a terse summary of fluxional analysis in Lemma 2, which Newton needed to treat the problem of an object falling through a resisting medium. In sum, Newton extended the scope of "geometry" in the Principia to handle a broad class of curves, leading to a much richer conception of geometry than that allowed by either Barrow or Descartes.

Following his remarks on the relationship between mechanics and geometry, Newton turns to giving a positive characterization of the basic problem of natural philosophy - "to discover the forces of nature from the phenomena of motions and then to demonstrate the other phenomena from these forces." Pursuing this project is possible because the trajectories generated by the interplay of inertia and the forces of nature can be determined exactly. The Principia offers an understanding of physical trajectories as generated by a combination of inertia and forces of nature that is analogous to that provided by a kinematical conception of geometrical curves as generated by a moving point or figure. Given this conception of rational mechanics as the project of discovering the generating forces for the trajectories of real objects, a number of questions about "forces of nature" are pushed to one side. For the inferential connection between the forces of nature and phenomena of motion, going in both directions, can be fully specified given a mathematical characterization of the force, without an account of its underlying source. (Although Newton in various places acknowledges the interest of determining the seat or cause of the forces of nature, an answer to such questions would not alter the Principia's project. Hence the causal question, which Barrow responded to by defending a version of formal causality, is left to one side.) Next I will turn to study a few aspects of the argumentative structure of the Principia that reflect Newton's response to the

11. Guicciardini provides a masterful overview of Newton's development of the calculus, which draws on the manuscripts and Whiteside's editorial apparatus in the MP; see also George E. Smith, "Newton's Research in Mathematics" (unpublished manuscript, 2005).

12. For discussions of the mathematical style of the Principia and Newton's reasons for adopting it, see D.T. Whiteside, “The mathematical principles underlying Newton's Principia Mathematica," Journal for the History of Astronomy 1 (1970): 116-138; MP 6; Michael S. Mahoney, "Algebraic vs. Geometric Techniques in Newton's Determination of Planetary Orbits," in Action and Reaction, ed. Paul Theerman and Adele F. Seeff (Newark, Delaware: University of Delaware Press, 1993), 183-205; and Guicciardini, Chapters 10-12. 
basic problem of philosophy. His response reflects the challenge of accessibility of the true motions: inferring the true motions and the forces responsible for them from the complex apparent motions of bodies we experience is a laborious and difficult, yet far from hopeless, undertaking.

\section{Handling Complexity in the Principia}

The Principia provides a framework for drawing inferences from observed trajectories to the underlying causes of motion, namely inertia and forces. This is analogous to analytical problems in geometry, in which a curve is given and the mechanism for generating the curve is to be found. In the first steps towards the Principia, taken in the manuscript De Motu, Newton treated the planetary trajectories, in effect, as given curves from which he inferred an inverse-square force law. If Newton had left it at that, the argument would have established the inverse-square force law for gravity in much the same way as earlier results had been established in Galilean-Huygensian mechanics: namely, that an inverse-square law produces trajectories with several striking features in common with observed motions. Even though the real motions are far too complicated for Kepler's laws to hold exactly, the account would be explanatory in much the same way as Galileo's treatment of projectile motion, despite its failure to account for air resistance.

It is a common mistake, however, to read the Principia as giving nothing more than a more elaborate version of this argument. Newton recognized the limitations of this initial argument and developed a sophisticated approach in the Principia to overcome them. ${ }^{13}$ There is a disanalogy between the analytic problem in geometry and the problem in natural philosophy: a trajectory is not "given" to the natural philosopher as the starting point of investigation, as it is to the geometer. The further steps Newton took in the Principia were driven in part by the challenge of reaching conclusions regarding real motions despite their enormous complexity. Rather than lowering the standard of success to require only qualitative agreement with the phenomena, Newton proved a number of results that allowed him to assess the impact of removing various idealizations.

\subsection{From De Motu to the Principia}

Christopher Wren offered Edmond Halley and Robert Hooke the reward of a a "forty-shilling book" for a proof that elliptical planetary trajectories follow from a force varying as the inverse square of the distance from the sun. Neither of them was up to the challenge, and Halley posed the problem to Newton on a visit to Cambridge in $1684 .{ }^{14}$ The brief manuscript Newton composed in response would have been sufficient to secure Newton a place in the history of mechanics.. ${ }^{15}$ The De Motu

13. Smeenk and Schliesser, in "Newton's Principia," in The Oxford handbook of the history of physics, ed. Jed Buchwald and Robert Fox (Oxford University Press, 2013), 109-165 gives a more thorough treatment of the structure of the Principia along these lines, which is particularly indebted to discussions of Newton's methodology with George Smith and his publications on the topic (George E. Smith, "The Newtonian Style in Book II of the Principia," in Isaac Newton's Natural Philosophy, ed. Jed Z. Buchwald and I. Bernard Cohen, Dibner Institute Studies in the History of Science and Technology [Cambridge, MA: MIT Press, 2001], 249-314; George E. Smith, "From the Phenomenon of the Ellipse to an Inverse-Square Force: Why Not?," in Reading Natural Philosophy: Essays in the History and Philosophy of Science and Mathematics to Honor Howard Stein on his 70th Birthday, ed. David B. Malament [Chicago: Open Court, 2002], 31-70; George E. Smith, "The Methodology of the Principia," in Cambridge Companion to Newton, ed. I. Bernard Cohen and George E. Smith [Cambridge: Cambridge University Press, 2002], 138-173).

14. There are two distinct problems: first, given the orbit or trajectory, find a force law sufficient to produce it, and, second, given the force law and initial position and velocity, determine the trajectory. It is not known precisely what problem Halley posed to Newton, but Newton's response addresses the first.

15. François de Gandt, Force and Geometry in Newton's Principia, trans. Curtis Wilson (Princeton: Princeton University Press, 1995). 


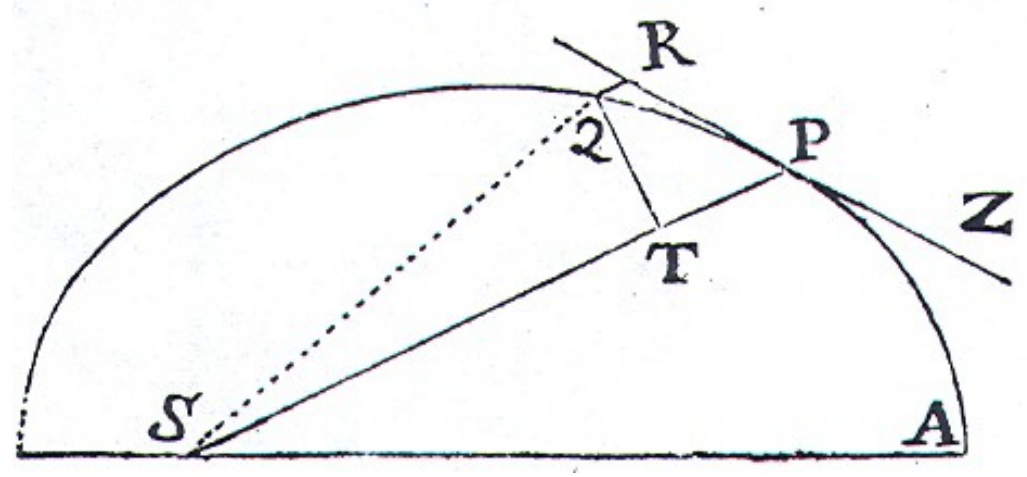

Figure 1: Figure from Proposition 6 in the Principia (and Theorem 3 of the De Motu).

achieved a unification of the Galilean-Huygensian theory of uniformly accelerated motion with Kepler's treatment of planetary motion, based on Newton's innovative treatment of force. But this was only the first step on the road to the Principia.

The central result of the De Motu brings together a generalization of Galileo's treatment of free fall with Kepler's area law, recognized as a general feature of motion under a central force (i.e., a centripetal force whose magnitude depends only on distance to the force center). Following Galileo, the distance traveled by a body starting at rest, undergoing uniform acceleration, is proportional to the square of the elapsed time. Newton realized that this proportionality holds for finite elapsed times in the case of uniform acceleration, but it is also valid instantaneously for arbitrary centripetal forces. A generalization of Galileo's result then provides Newton with a precise quantitative measure of a trajectory's deviation from straight, inertial motion at each point of the orbit given the magnitude of the force, which is allowed to vary: the deviation produced by any centripetal force is proportional to the square of the elapsed time, "at the very beginning of its motion". ${ }^{16}$ Newton next established, as his first Theorem, that what we now know as Kepler's area law holds for any central force: elapsed time of motion along the trajectory can be represented geometrically by the area swept out by a radius vector from the force center.

Combining these two results leads to a general expression relating the geometrical properties of the trajectory to the magnitude of the force and the law describing its variation. The deviation produced by the force acting at point $\mathrm{P}$ is represented by a line segment $\mathrm{QR}$, directed toward the force center (in Newton's figure, 3.1). This displacement is proportional to the product of the force $F$ acting on the body with the square of the time elapsed, $Q R \propto F \times t^{2}$, as shown by the generalization of Galileo's law. From Kepler's area law, $\mathrm{t} \propto \mathrm{SP} \times \mathrm{QT}$; it follows that $\mathrm{F} \propto \frac{\mathrm{QR}}{\mathrm{SP}^{2} \times \mathrm{QT}^{2}}$.

This theorem also illustrates how Newton extended classical geometry to treat instantaneous quantities, without explicit appeal to fluxional analysis. The result holds instantaneously, in the limit as the point $\mathrm{Q}$ approaches the point $\mathrm{P}$. The continuity of the generation of the curve guarantees, according to Newton, that a limiting value for the ratio of evanescent quantities exists, and it is given by the ratio of finite quantities. Enlarging the scope of geometry to include line segments of varying length allowed Newton to handle instantaneous quantities and the limiting behavior of their ratios. Newton used Theorem 3 by establishing connections between the "evanescent" figure QRPT and finite quantities characterizing the trajectory, such as the radius of a circle or the latus rectum of an ellipse. This leads to an expression for the force law entirely in terms of finite quanti-

16. This result appears in the Principia as Lemma 10, and the proof of the Lemma makes it clear that the proportionality only holds "ultimately" (or "in the limit" as the elapsed time goes to zero). 
ties, unblemished by evanescent quantities that vanish in the limit as $Q \rightarrow P$. Consideration of the figure QRPT thus allows Newton to handle the differential properties of the curve geometrically.

With these results, Newton generalized Huygens's earlier treatment of uniform circular motion to arbitrary curvilinear trajectories, and this opened the way for considering a variety of forces sufficient for motion along different plane curves. In particular Newton could directly address Wren's question. Halley et al. would have naturally wondered whether an inverse-square force sufficient for perfectly circular orbits would have to be supplemented by a secondary cause to account for elliptical motion. Newton's results show convincingly that a simple inverse-square force alone is sufficient for Keplerian motion. But more importantly, Newton concluded that planets held in their orbits by an inverse-square force directed at the sun move "exactly as Kepler supposed." Kepler's area law has special standing as a direct consequence of any central force, and Kepler's first and third laws hold for an inverse-square force, specifically. According to Kepler's first law, the planets follow elliptical trajectories. An inverse square force directed at the focus is sufficient to produce this motion if, in addition, the second law holds with respect to the focus of the ellipse (that is, the radius vector from the focus sweeps out equal areas in equal times). Thus, insofar as Kepler's laws hold exactly for each planet, one can infer an inverse square force between the sun and each of the planets. Kepler's third law is a specific instance of a general result linking periodic times to the exponent in the force law (Theorem 2). Furthermore, the ratio of the radii to the periods $\left(\mathrm{a}^{3} / \mathrm{P}^{2}\right)$ is the same for all of the planets, leading to the conclusion that a single inverse-square force directed at the sun suffices.

The Principia grew out of a number of questions provoked by De Motu. ${ }^{17}$ One line of thought forced Newton to abandon the simple picture of the planets following stable elliptical orbits, and the argument just summarized connecting these motions with a force law. The motion of the planets was apparently due to an inverse-square force directed at the sun, and similarly the motion of Jupiter's moons was due to an inverse-square force directed at Jupiter. How are these forces related? Such questions may have led Newton to apply what became the Principia's Third Law to combine the distinct forces due to the planets and the sun. Then the planets can no longer be described as responding to a single inverse-square force directed at a fixed sun, as there are other forces to take into account: the attraction of the planets on the sun, causing it to orbit a common center of gravity rather than remaining fixed, along with the interactions among the planets. Newton eloquently noted the consequences:

By reason of the deviation of the Sun from the center of gravity, the centripetal force does not always tend to that immobile center, and hence the planets neither move exactly in ellipses nor revolve twice in the same orbit. There are as many orbits of a planet as it has revolutions, as in the motion of the Moon, and the orbit of any one planet depends on the combined motion of all the planets, not to mention the action of all these on each other. But to consider simultaneously all these causes of motion and to define these motions by exact laws admitting of easy calculation exceeds, if I am not mistaken, the force of any human mind. ${ }^{18}$

Newton recognized the multifaceted challenge to reasoning from complex phenomena to the underlying forces, and Book 1 of the Principia lays the groundwork for Newton's response in Book

17. The manuscript was extended in a series of revisions leading to the Principia; see Herivel, in The Background to Newton's Principia (Oxford: Oxford University Press, 1965) and MP 6. Two other questions would likely have been raised by Halley: how do these ideas apply to the moon, with its apparently non-Keplerian motion, and to comets, which move through a much wider range of distances from the sun?

18. A. R. Hall and M. B. Hall, Unpublished Scientific Papers of Isaac Newton (Cambridge: Cambridge University Press, 1962), 280. 


\subsection{The Motion of Bodies}

How is one to proceed in the face of curves that no human mind can comprehend, as Newton so pointedly remarked? The results in Book 1 support a novel response to the challenges posed by the complexity of real phenomena. ${ }^{19}$ Newton's strategy proceeds in stages, beginning with a heavily idealized and tractable description of motion, and adding complexity as demanded by observations. I will emphasize three aspects of the Principia that are needed for this approach to be viable: first, the generality of Newton's treatment of force and trajectories; second, his handling of limiting relations to insure the durability of measurements; and, third, the controlled way in which he handles idealizations.

The generality of Newton's approach allows him to establish relationships between trajectories and force laws, rather than simply giving a collection of striking consequences for particular cases. Several theorems in Book 1 hold for arbitrary central forces. ${ }^{20}$ The force law itself is the unknown quantity to be determined based on the phenomena, so this level of generality is crucial. It leads to a much stronger inference, spanning a variety of alternative force laws rather than restricting consideration to a single force law (as in the De Motu).

The level of generality in the treatment of force is matched by the broad scope of curves Newton allows as possible trajectories. The collection of curves deemed intelligible by Descartes did not include a possible representation of the actual trajectory traced by Mars (idealized as a point mass), as a result of the complexity Newton acknowledged in the quote above. This problem would arise even for the motion of a pendulum bob, idealized again as a point mass, in a Huygensian cycloidal pendulum. Newton also proved (in Lemma 28) that there is no solution in terms of a Cartesian geometric curve to the so-called Kepler problem, which arises in finding the position of a planet as a function of time on a Keplerian ellipse. The solution required the use of what Newton called "geometrically irrational curves," which cannot be defined in terms of an equation with a finite number of terms. Hence celestial mechanics forces a conception of geometry broad enough to regard such curves as legitimate.

Proposition 1.45 illustrates the importance of both senses of generality; it shows how apsidal motion depends on the exponent of the power law of the underlying force. The apsides are the points of maximum and minimum distance from one focus of an elliptical orbit; in the case of apsidal precession, a body does not form a closed orbit and the apsides shift slightly with each revolution, by an amount given by the apsidal angle. The first corollary of 1.45 states that for nearly circular orbits if the apsidal angle $\theta$ is given by $n=\left(\frac{\theta}{\pi}\right)^{2}$, then the force is given by $f \propto r^{n-3}$. Based on this result, specific phenomena can be taken as measuring the parameters appearing in the force law, in the sense that there are law-like connections between the phenomena and parameters, within some delimited domain. ${ }^{21}$ Establishing such connections requires quantifying over a range of different force laws, which would not be possible without Newton's level of generality of force laws and trajectories. Furthermore, the relationship between the phenomena and the parameter

19. The Principia's first two books consider the motions of body generally, that is under a variety of different force laws, moving in spaces without resistance (Book 1) and with resistance of different kinds (Book 2). Book 3, "The System of the World," gives the argument for universal gravitation and further implications of gravity.

20. Most of the results are restricted to forces varying as $f \propto r^{n}$, for integer values of $n$, but several important theorems also hold for rational values of $n$ (e.g., Prop. 1.45).

21. Here I draw on the discussion of measurement given, in roughly this sense, by Harper, in Isaac Newton's Scientific Method: Turning Data into Evidence about Gravity and Cosmology (New York and Oxford: Oxford University Press, 2011), in his defense of a Newtonian ideal of empirical success. Cf. Dunlop, "The mathematical form of Measurement" for a different take on measurement, emphasizing connections with Barrow and the importance of judgments of congruence. 
of the force law is robust, in the sense that if the apsidal angle is approximately $\theta$, the force law is approximately $f \propto r^{n-3}$. With this proposition in hand, Newton can infer that the force law is approximately inverse square despite the complexity of the planetary orbits - it suffices to have established that they are very nearly elliptical with stable apsides.

This general treatment of force and trajectories makes it possible to regard observed motions as "measuring" parameters of the force law. This is obviously a heavily theory-dependent account of measurement. How stable are these results as one adds complexity, or changes the theory? Section 10 provides an example of how Newton responds to this concern, by considering the relation between earlier results regarding constrained motion, in particular Huygens's treatment of pendulums, and his own approach. ${ }^{22}$

Several lines of argument in the Principia depend upon using pendulums as measuring devices, and the case for universal gravity in particular relies on measurements of the local strength of surface gravity and its variation with latitude. Yet the claim that appropriately designed pendulums measure surface gravity depends, in Huygens's account, on a Galilean conception of gravity, which treats gravity as uniform acceleration directed along parallel lines. Rather than simply making an argument that Huygens's results are a useful approximation to universal gravity, Newton proves theorems that specify a precise limiting relationship (although Newton does not explicitly state it in these terms): Galilean gravity holds in the asymptotic limit of universal gravity at or below the surface of a uniformly dense spheroid as the ratio of any vertical distance of interest $d$ to the radius of the earth $R$ goes to zero, $\frac{d}{R} \rightarrow 0 .{ }^{23}$ This insures that the relationships between physical quantities asserted in Huygens's theory retain approximate validity. At or below the surface of a uniformly dense spheroid, Huygens's results still describe law-like relationships among quantities mirroring the law-like relationships within Newton's theory. This claim is restricted to a specific domain, but it is the domain for which Newton needed to establish that pendulums could be used as measuring devices.

The results in Section 10 thus allow Newton to argue that Huygens had successfully measured the time of free fall, not because of his adoption of Galilean gravity as the underlying theory, but in spite of it. In the domain Huygens considered, the physical relationships asserted by the superseded theory of Galilean gravity approximately match those of Newton's own theory. Hence the measurements of surface gravity - and, indeed, the entire body of evidence in favor of Galilean-Huygensian mechanics - could be subsumed within Newton's theory. Evidence consisting of theory-dependent measurements can be durable enough to survive transitions in underlying theory.

Turning to the third point, Newton notes that the first 10 sections of Book 1 concern "bodies attracted toward an immovable center, such as, however, hardly exists in the natural world" (Principia, 561). Since gravity is a mutual interaction, it is unphysical to regard one body as fixed instead, a system of interacting bodies will orbit a common center of gravity. It is also unphysical to treat these interacting bodies as if they are point masses, rather than having finite extent. The force the Earth exerts on a nearby body is composed out of the attractive forces on each part of the body from each part of the Earth. Newton realized in composing the Principia that the resultant force may not have a simple form even if the component forces do. Newton's predecessors often acknowledged similar limitations, then argued that the theory nonetheless provides an approximate description of the world. Newton's response is strikingly different, as he considers in detail the

22. This brief discussion of Section 10 summarizes the conclusions of Smeenk and Smith, in "Newton on Constrained Motion" (Unpublished manuscript, 2012).

23. The restriction to a uniformly dense spheroid is needed to insure that gravity inside the spheroid varies directly with distance. This relationship is formulated with regard to a specific physical situation. The Galilean theory does not approximate Newton's theory in all cases, and the asymptotic limit is needed because in Newton's theory the case described by Galilean gravity only obtains in the limit and not in any physically realizable case. 
consequences of relaxing both of these simplifying assumptions.

Newton obtained limited results for systems of interacting bodies in Section 11. A number of results regarding bodies orbiting a fixed force center carry over directly to the case of two interacting bodies. In the three body case for an inverse square force, Newton's results consisted of a series of corollaries describing various effects in a three-body system (such as the Earth-moon-Sun system) in qualitative terms. Although Newton regarded these as "imperfect," they indicate various consequences of treating the mutual interactions among all three bodies. Sections 12-13 consider bodies of finite extent, and Newton established a remarkable feature of an inverse-square force law. For if we idealize the Earth as a spherical body whose density varies with the radius, the total force on a nearby body will be an inverse-square force directed at the center of the Earth with the absolute measure of the force given by the total mass. He further proved the famous result that two spherical bodies interacting via an inverse-square force can be treated as mass points interacting with forces depending on their total masses directed at their respective centers.

These three sections reveal a quite sophisticated approach to handling idealizations. ${ }^{24}$ Rather than treating his initial account as a good approximation despite its unphysical idealizations, Newton developed the mathematics needed to assess the effects of removing the idealizations. The initial account is itself exact, given a precise quantitative treatment, yet acknowledged to differ from the true trajectory. Newton's results, while incomplete, could be used to characterize qualitatively the departures from the initial idealized treatment due to many-body interactions and the finite extent of real bodies. These results made it possible to identify the kind of contrasts between theoretical calculations and observation one would expect to see as a result of specific idealizations, and to assess whether removing a particular idealization would lead to an exact trajectory closer to the true trajectory. Many of the effects identified in these sections were already relevant to assessing the application of the theory to the solar system, treated in Book 3.

Together these three aspects of Book 1 support an approach to the complexity of real motions that proceeds in successive stages, dubbed the "Newtonian style" by Cohen. ${ }^{25}$ My characterization of this style follows Smith's much richer account in the references cited above. At a given stage the physical trajectories will be treated as if they were produced by an explicit combination of forces. The resulting exact trajectory is expected to differ from the true trajectory, because the derivation, to be tractable, will include a number of presumably false idealizing assumptions. Even so, the idealizations can be used in making inferences concerning values of physical quantities as long as these inferences are robust, as in the case of Prop. 1.45. This inference holds even if the physical trajectory is only approximately an elliptical orbit with stable apsides. Of course, this inference clearly depends upon the basic Newtonian framework and the earlier stages of the description of the system. Newton showed, however, that in the specific case of Huygens's pendulum measurements conclusions based on measurements in this sense can be durable through theory change.

The demand for exactness at each stage of inquiry also lends particular significance to discrepancies between the calculated trajectories and observations. Results like those in Sections 11-13 put Newton in a position to assess whether particular systematic deviations from idealized models can be eliminated by dropping specific assumptions and developing a more complicated model. If the empirical deviations from the ideal case are of this kind, then the research program can proceed by relaxing the idealization. But it is also possible to identify systematic deviations that instead reveal deeper problems with the entire framework of Book 1, exemplified by Mercury's anomalous perihelion motion. Newton's treatment of idealizations allows for observations to continue to guide research, even though the identification of the deviations in question presupposes that the simplest

24. Smith, "The Newtonian Style in Book II of the Principia"; Smith, "The Methodology of the Principia."

25. I.B. Cohen, The Newtonian revolution: with illustrations of the transformation of scientific ideas (Cambridge University Press, 1980). 
idealized models are approximately correct.

\subsection{Arguing from the Phenomena}

The body of results in Book 1 provided Newton with mathematical tools to infer the underlying forces of nature from the phenomena of motion. Nature also cooperated in providing a system of bodies that could be described to a very good approximation as interacting via a single force, whose motion had long been studied and described with obsessive precision. In the opening sequence of Book 3 Newton argues from observed regularities of the motion of the planets and their satellites to the properties of the force of gravity. Here is not the place to review that famous argument (see, in particular, Harper, Isaac Newton's Scientific Method), but I will briefly contrast it with the argument in the De Motu.

Based on the results of the De Motu, Newton could have claimed that idealized descriptions of bodies moving in response to gravity agree qualitatively with observed planetary motions. Newton had shown that Kepler's laws hold for bodies moving in response to an inverse-square force directed at the sun, regarded as fixed. Yet there are two shortcomings of an argument based on these results. First, the inference presupposes that the planet moves exactly on an ellipse with a force directed at the sun at one foci; the argument does not apply to nearly elliptical trajectories or forces directed nearly at one foci. ${ }^{26}$ Once Newton considered the effect of mutual interactions, he realized that the planets do not follow exactly elliptical trajectories - blocking the De Motu argument. Second, there are challenges regarding the status of various idealizations that could not be addressed. Is it legitimate, for example, to treat Mars and the Sun as point-masses in calculating their trajectories, and to neglect the gravitational attraction Mars exerts on the Sun?

In the Principia Newton overcame both of these shortcomings. The opening sequence of Book 3 gives an argument for universal gravitation that is robust, in the sense that the inferences do not require that the antecedent holds exactly in order to reach conclusions regarding the force law (as in Prop. 1.45). Much of the remainder of Book 3 is devoted to discussing the effects of dropping various idealizations to give a more realistic description, e.g. in studying the Earth-Moon-Sun system. These are the kinds of questions that the results discussed above put Newton in a position to answer, albeit provisionally.

The further results in Book 3 embodied a distinctive approach to problems in celestial mechanics that would set the agenda for the eighteenth century. ${ }^{27}$ Newton treated celestial motions as consequences of the gravitational interactions among a system of bodies. The goal at any given stage of inquiry would be to provide an exact description of the motions that would result given several idealizations. This exact trajectory could then be compared with observations. At each stage the theoretical description was well controlled in the sense that the idealizations needed to derive a specific solution were explicitly identified. Any systematic discrepancies could then be used to identify physically significant features that had been initially excluded in the description. Finally, Newton's further results indicating how the behavior of the system changes as a result of dropping particular idealizations provided the basis for distinguishing troubling discrepancies from

\footnotetext{
26. Newton had proved that for a body moving on an elliptical trajectory with the force directed at the center, the force varies directly with the distance. Yet for elliptical orbits that are nearly circular, as in the case of the planets, the two foci and the center are not far apart. As Smith, in "From the Phenomenon of the Ellipse to an Inverse-Square Force: Why Not?" discusses, an inference to an inverse-square force from Kepler's first law is not robust, in the sense that the conclusion does not follow if the antecedent only holds approximately.

27. See Wilson, in "The Newtonian achievement in astronomy.," in Planetary Astronomy from the Renaissance to the Rise of Astrophysics. Part A: Tycho Brahe to Newton, vol. 1 (1989), 233-274, Smith, in "Closing the Loop," in Newton and Empiricism, ed. Zvi Biener and Eric Schliesser (Oxford: Oxford University Press, 2014), 262 for more detailed discussions of Newton's contribution to celestial mechanics.
} 
those that one could expect to be handled at the next stage of theoretical refinement. Insofar as one encounters only discrepancies of the latter sort, it is plausible to take the understanding of the physical trajectory as generated by the forces explicitly taken into account by that stage as very nearly accurate, albeit not exact. One can imagine approaching a completely exact description of the physical trajectory, with a fully specified account of the forces generating the motion, as a kind of limit.

This should not be regarded, however, as approaching a closer and closer match with a preexisting description of the true trajectory. The projects of developing a more detailed theoretical account of the motion and of giving a more exact observational characterization often went progressed in tandem. In many cases, without the structure provided by the theoretical skeleton, observations would remain a complex, amorphous mass.

The problem of the moon exemplifies the importance of Newton's approach. Seventeenth century astronomers struggled to describe the moon's motion with accuracy comparable to that achieved for the planets. In order to assess whether the details of the moon's motion were compatible with gravitational theory, Newton had to first develop a more accurate description of the motion itself, and he tackled the theoretical and observational problems simultaneously. The physical trajectory only becomes intelligible via a combination of observations and a theoretical understanding of how it is generated. Newton's aim was to account for the various known inequalities in the lunar orbit as a consequence of the perturbing effect of the Sun's gravity and other features of the EarthMoon-Sun system. He was ultimately not able to make substantial progress with regard to accuracy over the ideas of Jeremiah Horrocks (from the 1630s). His approach to the problem proved to be more influential. His $18^{\text {th }}$ century successors were able to employ new mathematical methods that made it possible to enumerate all of the perturbations at a given level of approximation, namely as all of the terms at a given order in an analytic expansion. It was only with a more sophisticated mathematics that astronomers could fully realize the advantages of approaching the complexities of the moon's motion via a series of approximations. Although it drew on tools unavailable to Newton, this work followed Newton's approach to achieving high levels of precision by using a series of well-controlled idealizations.

\section{Mathematization and Certainty}

Newton directly addressed the contribution mathematics makes to achieving the "highest evidence" for claims in natural philosophy in only a few places in his published work; in addition to the Principia's preface, the most detailed discussion appears in Query 31 of the Opticks (1717). Newton's main concern was to characterize his natural philosophy in terms of analysis and synthesis, as part of a defense of his methodology and its fruits in reply to continental critics. As Domski ${ }^{28}$ has emphasized, in this and similar passages Newton locates his methodology in a historical tradition, as a revival and extension of ancient mathematical practice. This concern is nearly orthogonal to questions regarding the epistemology of geometry that are our main focus. Guicciardini, Chapter 14 provides a thorough exegesis of Query 31 and related passages, drawing on a careful assessment of analysis and synthesis in Newton's mathematical practice. Yet, as Guicciardini acknowledges, these passages are not sufficient to elucidate Newton's philosophy of geometry. Hence, I will turn to an unpublished manuscript customarily called "De Gravitatione" (hereafter, DG) rather than Newton's sparse methodological remarks. Passages in the DG suggest that Newton regarded the certainty of geometry as grounded in knowledge of the nature of space. For the metaphysical account of space

28. Domski, "Newton as Historically-Minded Philosopher." 
to play such a role, we must have access to its structure. Here I will briefly consider the epistemology of geometry suggested in the DG, and argue that in the Principia geometry becomes entangled with dynamics.

Much of DG is devoted to a detailed critique of Cartesian views regarding space, body, and motion. Newton argued, contra the Cartesian identification of space and body, that an adequate definition of motion had to be formulated in terms of "some motionless being such as extension alone, or space in so far as it is seen to be truly distinct from bodies." ${ }^{29}$ His ensuing discussion of the ontological status of this distinct entity and its properties sheds some light on the status of geometry. The object of geometry is clearly space itself, and not, by contrast with Barrow, properties of objects. Barrow held that were "the Hand of an Angel" to polish a solid particle of matter, a perfectly spherical surface would be revealed. Sphericity is a property of a particle of matter, rather than being a merely mental entity or having a distinctive mode of being. Newton also rejected the latter two options, but ascribed geometrical properties to space rather than bodies:

For thus we believe all those spaces to be spherical through which any sphere ever passes, being progressively moved from moment to moment, even though a sensible trace of that sphere no longer remains there. We firmly believe that the space was spherical before the sphere occupied it, so that it could contain the sphere; and hence as there are everywhere spaces that can adequately contain any material sphere, it is clear that space is everywhere spherical. ${ }^{30}$

Newton attributed geometrical properties to all regions of space. He further argued that bodies bear geometrical properties derivatively, as "determined quantities of extension" endowed with additional attributes, such as impenetrability. Finally, the geometrical structure ascribed to space itself, along with an independent structure relating locations at different times, is necessary, as Newton argued in a famous rebuttal of Descartes, for an adequate definition of motion. ${ }^{31}$

Some of Newton's remarks in DG suggest a straightforward empiricist conception of geometry. The main challenge facing such an account, as Torretti succinctly put it, is that "geometrical objects [...] are nowhere to be found in experience exactly as geometry conceives them". ${ }^{32}$ Barrow and Newton alike regarded geometry as treating properties of real entities - bodies and space itself, respectively - that are not directly present in experience. How then does experience bear on the concepts and theorems of geometry? Barrow's position seems to be that experience can establish the existence of geometric properties. Any actual line is not straight, for example, but we can see that it can always be straightened, leading to a geometric line as a limit. It is unclear exactly how experience reveals that this refinement of our experienice is a real possibility. ${ }^{33}$ Newton held that space itself is insensible, so its structure must be discerned based on the geometric properties of objects. We obtain the idea of space from experience by abstracting away dispositional and sensible features of bodies, leaving us with an "exceptionally clear idea of extension," namely "the uniform and unlimited stretching out of space in length, breadth and depth." 34 In this context, the question analogous to that confronting Barrow regards how the character of sense experience justifies this "clear idea" and the six properties of space Newton goes on to elucidate. Taking bodies as the

29. Isaac Newton, Isaac Newton: Philosophical Writings, ed. A. Janiak (Cambridge: Cambridge University Press, 2004), 20-21.

30. Ibid., 23.

31. For discussions of this argument, see the sources cited in footnote 6.

32. Roberto Torretti, Philosophy of geometry from Riemann to Poincaré (Dordrecht: D. Reidel, 1978), p. 254.

33. See Dunlop, in "What geometry postulates" for a sympathetic reconstruction of Barrow's appeal to practice as a response to this question.

34. Newton, Isaac Newton: Philosophical Writings, p. 22. 
basis for abstraction is particularly problematic given the status of Newton's account of body in DG: he presented it as sufficient to ground our sense experience, but did not claim that it is the only such account. ${ }^{35}$ Such an uncertain account of body cannot provide firm foundations for geometric knowledge, and in any case Newton's arguments do not follow Barrow's approach in appealing to abstraction and refinement of our experience of bodies.

Newton's argument for the infinity of space, the second property he discusses at length, takes a quite distinctive approach: it depends on a simple geometric construction, carried out in the imagination. This appeal to the imagination, as a form of access to truths about the nature of space, marks a further critical response to Descartes. ${ }^{36}$ Descartes rejected the scholastic idea that knowledge of geometry is derived from sense experience via a process of abstraction, and instead held that geometry states claims regarding the true and immutable natures of geometric objects. The eternal and immutable natures exist, in some sense, as innate ideas in created minds. Newton clearly did not adopt this Cartesian approach fully; in a later unpublished manuscript intended as a fifth rule for philosophizing (to be added to those in the Principia), probably reflecting Locke's influence, Newton explicitly rejected the possibility of innate ideas. ${ }^{37}$ Yet the argument for the infinity of space is based on the ability to imagine the following construction (cf. Domski, "Newton and Proclus"). Consider opening up a given triangle by rotating one of its sides around a vertex, with the other sides fixed, and defining a sequence of points where the line segment extending the rotated side meets a line segment extending the opposite fixed side. There is no final point of this sequence; Newton concluded that the real line defined by the sequence is longer than any finite length. The contrast with Descartes is clear, regarding both the strength of this conclusion (namely, that space is infinite rather than merely of indefinite extent, as Descartes held) and the roles of imagination and understanding. Newton further argued that we can understand the infinity of space positively rather than merely as a lack of boundaries, as illustrated by geometrical objects with finite surface areas and infinite lengths. ${ }^{38}$ The later manuscript "Tempus et Locus" 39 includes a wide variety of other examples illustrating our ability to comprehend infinite quantities in mathematics.

It is challenging to find a coherent epistemology of geometry in the DG. Although I will not argue the point here, on my reading there are unresolved tensions among the lines of argument Newton gives regarding the nature of space. ${ }^{40}$ These tensions can be avoided to some extent because the different properties of space Newton considers are nearly independent of one another. The infinity of space does not determine the other local features Newton identifies, such as Euclidean geometrical structure ascribed directly to space, and the relation among spaces at different times needed to define motion.

35. See McGuire, in "A dialogue with Descartes: Newton's ontology of true and immutable natures," Journal of the History of Philosophy 45, no. 1 (2007): $\S 6$ and Stein, in "Newton's Metaphysics," in Cambridge Companion to Newton, ed. I. Bernard Cohen and George E. Smith (Cambridge: Cambridge University Press, 2002), 256-307.

36. See, in particular, McGuire, "A dialogue with Descartes" for an assessment of Newton's debt to, and contrasts with, Descartes, as well as Domski, in "Newton and Proclus: geometry, imagination, and knowing space," The Southern Journal of Philosophy 50, no. 3 (2012): 389-413, who draws interesting parallels with Proclus's neo-Platonic philosophy of mathematics, and Stein, in "Newton's Metaphysics". See also Biener and Smeenk, in “Cotes' Queries: Newton's Empiricism and Conceptions of Matter," in Interpreting Newton: Critical Essays, ed. Andrew Janiak and Eric Schliesser (Cambridge: Cambridge University Press, 2012), 105-137 for a discussion of the account of body in DG parallel to this discussion of space.

37. Alexandre Koyré, Newtonian Studies (Cambridge, MA: Harvard University Press, 1965), 272.

38. Newton probably had cases such as Torricelli's trumpet in mind, which Barrow discussed in Lecture 16 of his Mathematical Lectures.

39. Dated to the 1690s, and published with translation, by JE McGuire, "Newton on place, time, and God: An unpublished source," British Journal for the History of Science 11 (1978): 114-129.

40. But see Domski, "Newton and Proclus," for the most persuasive and careful attempt at a coherent account. 
More importantly, however, I regard DG as a transitional text in which Newton had not fully developed the insights regarding dynamics that are crucial to the Principia. The accounts of body, space, and time, and the relations among them, differ substantively in the Principia and in later texts such as "Tempus et Locus." ${ }^{11}$ Far from providing philosophical foundations for the account of space and time in the Principia, as Domski ${ }^{42}$ argues, DG was written prior to Newton's recognition of the importance of specifying particular measures of space and time and the full implications of the relativity of motion. There are only hints of Newton's innovative conceptions of force and mass in $\mathrm{DG},{ }^{43}$ and developing these ideas led to distinctive account of geometry reflected in the Principia's preface and related texts discussed in $\S 2$. The considerations of measurement and dynamics that are central to the Principia lead to a physical conception of geometry. On this account, the geometrical properties of space are related to sense experience only quite indirectly, by virtue of their role in a dynamical account of motion.

The Principia starts with the phenomena of motion, and our observations of the trajectories of bodies over time. All of our descriptions of motion are implicitly made with respect to some relative space - for example, the motion of balls dropped from the dome of St. Paul's Cathedral (in one of Newton's experiments) were described relative to the building, with times determined by a pendulum clock. An initial description of the trajectory of the body could start by assigning spatial dimensions with respect to this relative space; obviously, the trajectory will generally not be the same with respect to a different relative space. In formulating the relativity of motion in Corollaries 5 and 6, Newton recognized that some specific choices of relative space do not lead to any dynamical differences in the descriptions of the motion; in anachronistic language, there is an equivalence class of relative spaces that give dynamically equivalent descriptions. There are also relative spaces that do lead to dynamical differences. If the relative space used to describe these motions is itself rotating, as St. Paul's does due to the motion of the Earth, the true forces arising as interactions among bodies will not match the observed accelerations (due, in this case, to the Coriolis force). In principle, once such an acceleration is discovered one could describe the motion more accurately using a different relative space (in this case, by acknowledging Earth's motion), with the hope of eventually determining the true motions. At the end of the famous scholium on space and time, Newton remarks that the entire Principia was composed so that the true motions could be found, and that "the situation is not entirely desperate" (Principia, 414). A relative space that is not suitable should always be revealed, in principle, by bodies accelerating without an identifiable physical force, but isolating such an effect requires first identifying and characterizing all the other forces relevant to the bodies' motion - and doing so requires employing the full framework of the Principia. The results described in $\S 3$ above provide the framework to carry out a study of this kind for real motions, such as the motion of the objects in the solar system. Newton's success in characterizing a single force responsible for all of these motions, despite their complexity, makes a compelling case against desperation, in the sense that the study of trajectories within the quantitative framework of the Principia suffices to establish the underlying dynamics.

Geometrical ideas enter into this description of motion in two different senses. First, there are the measurements of geometrical properties and congruence made with respect to a chosen relative space, which are refinements of our subjective experience of distances and times. Geometry in this sense is not tied to the structures of "absolute space" described in DG, because the relationship between the chosen relative space and absolute space is unknown. Absolute space is itself insensible, as Newton remarks (Principia, 414), and the connection between a given relative space and abso-

41. There is much more continuity in other aspects of Newton's views that are not my focus here, such as the infinity of space and its relation to God.

42. Domski, "Newton and Proclus."

43. Biener and Smeenk, "Cotes' Queries.” 
lute space arises only at the end of inquiry, so to speak - corresponding to a stage of inquiry where all accelerated motions have been attributed to a physical force, with no discrepancies. Despite the inaccessibility of absolute space, it plays a fundamental role in Newton's dynamical analysis of motions: the crucial distinction between accelerated and non-accelerated motion cannot be adequately captured, as Newton famously argued in the Scholium, in terms of motion with respect to relative spaces. Second, geometry enters directly into the formulation of the dynamics, as part of the characterization of the force law. The force of gravity, for example, depends on the distances among interacting bodies. Yet the geometrical structure required to fulfill this role is evanescent, in the sense that the force law depends only on the configuration of bodies at a given instant. ${ }^{44}$ This second sense of spatial geometry connects back to the first via a dynamical account of how spatial measurements can be performed - for example, by using rigid bodies that can be moved from one location to another in order to make assessments of congruence. The subtlety of this connection between the dynamical role of geometry and geometrical properties revealed by measurements follows from the relativity of motion. The instantaneous geometrical properties may be shared with respect to different relative spaces, yet comparisons of locations at different times, as will be required for any spatial measurements, implicitly depend on a specific choice of relative space.

\section{Conclusion}

In closing, let me return to the general theme of the mathematization of nature by reflecting on the sense in which Newton's natural philosophy is based on mathematical principles. I have argued that Newton rejected one objection to this idea as simply reflecting a confusion: mechanics is no less exact in describing its objects of study than geometry. This is particularly clear given his kinematic conception of curves and its close parallel with the mechanical generation of curves via forces. Yet in order to maintain that geometry studies properties of material rather than abstract entities, Newton is forced to regard these properties as remote from direct sensory experience. The main challenge is then one of epistemic accessibility: granted that there is an underlying quantitative structure that it is the aim of natural philosophy to uncover, how can this be done? The most striking aspect of the Principia the depth of Newton's insight and mathematical resourcefulness in responding to this problem. The Principia's mathematical framework provided a way of reasoning from the evidence provided by observed trajectories to claims about the underlying dynamics. The use of a mathematical framework to implement research via a controlled sequence of successive approximations is Newton's most influential contribution to the mathematization of nature within physics. The object of geometry, absolute space, is pushed to lie beyond our possible sense experience, as an ideal limit that may be reached only at the end of the series of successive approximations. It is then no surprise that Kant identified the nature and status of our knowledge of geometry as a pressing foundational problem for Newtonian science.

44. For further discussion, see in particular, Howard Stein, "On relativity theory and openness of the future," Philosophy of science 58, no. 2 (1991): 147. 


\section{References}

Barrow, Isaac. The Usefulness of Mathematical Learning Explained and Demonstrated: Being Mathematical Lectures Read in the Publick Schools at the University of Cambridge. London: Stephen Austen, 1734.

Biener, Zvi, and Chris Smeenk. "Cotes' Queries: Newton's Empiricism and Conceptions of Matter." In Interpreting Newton: Critical Essays, edited by Andrew Janiak and Eric Schliesser, 105-137. Cambridge: Cambridge University Press, 2012.

Bos, H.J.M. Redefining geometrical exactness: Descartes' transformation of the early modern concept of construction. New York: Springer Verlag, 2001.

Cohen, I.B. The Newtonian revolution: with illustrations of the transformation of scientific ideas. Cambridge University Press, 1980.

Dijksterhius, E. J. The Mechanization of the World Picture. Paperback (1986). Translated by C. Dikshoorn. Princeton University Press, 1961.

DiSalle, R. Understanding space-time: The philosophical development of physics from Newton to Einstein. Cambridge: Cambridge Univ Press, 2006.

Domski, M. "Newton and Proclus: geometry, imagination, and knowing space." The Southern Journal of Philosophy 50, no. 3 (2012): 389-413.

. "Newton as Historically-Minded Philosopher." In Discourse on a New Method: Reinvigorating the Marriage of History and Philosophy of Science, edited by M. Domski and M. Dickson, 65. Open Court Pub Co, 2010.

- "The constructible and the intelligible in Newton's philosophy of geometry." Philosophy of Science 70, no. 5 (2003): 1114-1124.

Dunlop, Katherine. "The mathematical form of measurement and the argument for Proposition I in Newton's Principia." Synthese 186, no. 1 (2012): 191-229.

- "What geometry postulates: Newton and Barrow on the relationship of mathematics to nature." Interpreting Newton: Critical essays (2012): 69-101.

Earman, John S. World Enough and Space-Time: Absolute versus Rational Theories of Space and Time. Cambridge, MA: MIT Press, 1989.

Gandt, François de. Force and Geometry in Newton's Principia. Translated by Curtis Wilson. Princeton: Princeton University Press, 1995.

Guicciardini, N. Isaac Newton on mathematical certainty and method. The MIT Press, 2009.

Hall, A. R., and M. B. Hall. Unpublished Scientific Papers of Isaac Newton. Cambridge: Cambridge University Press, 1962.

Harper, William L. Isaac Newton's Scientific Method: Turning Data into Evidence about Gravity and Cosmology. New York and Oxford: Oxford University Press, 2011.

Herivel, J. The Background to Newton's Principia. Oxford: Oxford University Press, 1965.

Jesseph, Douglas. Squaring the circle: the war between Hobbes and Wallis. Chicago: University of Chicago Press, 1999. 
Koyré, Alexandre. Newtonian Studies. Cambridge, MA: Harvard University Press, 1965.

Mahoney, Michael S. "Algebraic vs. Geometric Techniques in Newton's Determination of Planetary Orbits." In Action and Reaction, edited by Paul Theerman and Adele F. Seeff, 183-205. Newark, Delaware: University of Delaware Press, 1993.

"Barrow's mathematics: between ancients and moderns." Before Newton: The Life and Times of Isaac Barrow (1990): 179-249.

Mancosu, Paolo. Philosophy of mathematics and mathematical practice in the seventeenth century. Oxford University Press, 1996.

McGuire, JE. “A dialogue with Descartes: Newton's ontology of true and immutable natures." Journal of the History of Philosophy 45, no. 1 (2007): 103-125.

—. "Newton on place, time, and God: An unpublished source." British Journal for the History of Science 11 (1978): 114-129.

Newton, Isaac. Isaac Newton: Philosophical Writings. Edited by A. Janiak. Cambridge: Cambridge University Press, 2004.

The Principia, Mathematical Principles of Natural Philosophy: A New Translation. Translated by I. Bernard Cohen and Anne Whitman. Berkeley, California: University of California Press, 1999 [1726].

Rynasiewicz, R. "By their properties, causes and effects: Newton's scholium on time, space, place and motion-I. The text." Studies In History and Philosophy of Science Part A 26, no. 1 (1995): 133-153.

Smeenk, Chris, and Eric Schliesser. "Newton's Principia." In The Oxford handbook of the history of physics, edited by Jed Buchwald and Robert Fox, 109-165. Oxford University Press, 2013.

Smeenk, Chris, and George E. Smith. "Newton on Constrained Motion." Unpublished manuscript, 2012.

Smith, George E. "Closing the Loop." In Newton and Empiricism, edited by Zvi Biener and Eric Schliesser, 262. Oxford: Oxford University Press, 2014.

"From the Phenomenon of the Ellipse to an Inverse-Square Force: Why Not?" In Reading Natural Philosophy: Essays in the History and Philosophy of Science and Mathematics to Honor Howard Stein on his 70th Birthday, edited by David B. Malament, 31-70. Chicago: Open Court, 2002.

. "Newton's Research in Mathematics." Unpublished manuscript, 2005.

. "The Methodology of the Principia." In Cambridge Companion to Newton, edited by I. Bernard Cohen and George E. Smith, 138-173. Cambridge: Cambridge University Press, 2002.

- "The Newtonian Style in Book II of the Principia." In Isaac Newton's Natural Philosophy, edited by Jed Z. Buchwald and I. Bernard Cohen, 249-314. Dibner Institute Studies in the History of Science and Technology. Cambridge, MA: MIT Press, 2001.

Stein, Howard. “Newtonian space-time.” Texas Quarterly 10 (1967): 174-200.

"Newton's Metaphysics." In Cambridge Companion to Newton, edited by I. Bernard Cohen and George E. Smith, 256-307. Cambridge: Cambridge University Press, 2002. 
Stein, Howard. "On relativity theory and openness of the future." Philosophy of science 58, no. 2 (1991): 147.

Stewart, Ian. "Mathematics as Philosophy: Proclus and Barrow." Dionysius 18 (2000): 151-181.

Torretti, Roberto. Philosophy of geometry from Riemann to Poincaré. Dordrecht: D. Reidel, 1978.

Whiteside, D.T., ed. The Mathematical Papers of Isaac Newton. Cambridge, London: Cambridge University Press, 1967-81.

. "The mathematical principles underlying Newton's Principia Mathematica." Journal for the History of Astronomy 1 (1970): 116-138.

Wilson, C. "The Newtonian achievement in astronomy." In Planetary Astronomy from the Renaissance to the Rise of Astrophysics. Part A: Tycho Brahe to Newton, 1:233-274. 1989. 